\title{
Recent trends on mobile computing and future networks
}

\author{
Kyung-Yong Chung $\cdot$ Junseok Yoo $\cdot$ Kuinam J. Kim
}

Published online: 1 May 2013

(c) Springer-Verlag London 2013

With the development of IT convergence technologies to improve quality of life, users can now more easily access useful information. A convergence system represents an environment that is able to provide personal services by configuring various devices and sensors based on both wire and wireless networks. Further, diverse and far-reaching information is being produced fast and distributed instantly in digitized format. Studies on mobile computing are continuously presenting more efficient ways of delivering information to more users. Mobile computing is a technology that provides a service automatically based on perceived situational information in personal and ubiquitous environments. Ubiquitous computing is characterized by users who are focused on a virtual space established by computers and networks. However, mobile computing groups of computers work through various sensors that exist in the real world. Users are able to receive various personal services using many different types of mobile computing resources within an internal/external space, without limitations in time or space [1,2]. Previously, users had to convey their intentions using standard input devices and obtain the results on an output device. On the contrary,

K.-Y. Chung ( $₫)$

Department of Computer Information Engineering,

Sangji University, Wonju, Republic of Korea

e-mail: dragonhci@gmail.com

J. Yoo

Institute for Creative and Advanced Technologies,

Suwon, Republic of Korea

e-mail: junsyoo@gmail.com

\section{K. J. Kim}

Department of Industrial Security, Kyonggi University,

Suwon, Republic of Korea

e-mail: harap123@hanmail.net in a distributed and mobile computing environment, life$\log$, sensors, big data, and computing resources are ubiquitous in a user's everyday life. To provide personal services according to various lifestyles, these computing resources should be aware of a user's intentions and the surrounding environment, as well as provide optimal services $[3,4]$.

- Mobile Applications and Convergence System

- Mobile, Wireless, Wired Convergence

- Mobile Convergence Service Architecture

- Convergence Networks and Communications

- Convergence Personal Communication

- Convergence Mobile Technology

- Intelligent Information Extraction

- Convergence Information Indexing, Searching, and Visualization

- Access Control and Privacy Protection in Pervasive Computing

- Personal Healthcare Services

This special issue has become one of the hottest topics in mobile computing and future networks. We believe that this special issue will have a high citation in the areas of personal and ubiquitous computing.

A study by Lee et al. presented an IKEv2 authentication exchange model and performance analysis in mobile IPv6 networks. They designed and constructed a network based on real mobile nodes, modeled an IKEv2 protocol through the simulation, analyzed authentication key resetting and rekeying of the IKEv2 protocol in a MIPv6 network, and experimented with the effects of limited bandwidth on key exchange with analysis data. As a result of the experiment, it was confirmed that the rekeying value was a little faster than initializing, and then, the router disconnection was recognized as the handover process and performed 
resetting and rekeying. This will be available for secure transmission for heterogeneous layers to be exchanged through a multi-interface.

A second paper by Park et al. introduced an integrated model to analyze driver acceptance of car navigation systems by incorporating potential relationships with possible psychological factors on a technology acceptance model (TAM). Among the proposed measures, the TAM, introduced by Davis, is one of the most widely used and successful model at elucidating how people accept a particular system. This study indicated that the effects of PLA and PPS on PU, SDQ, and ATT are significant. Considering that PU and SDQ are two of the main determinants of driver acceptance of car navigation systems, they should be further considered in order to provide higher usability and quality of systems to respond to users' needs.

A third paper by Kang et al. presented head detection and tracking systems for visual surveillance. They utilized a hidden Markov model for more accurate recognition and to absorb the few errors that can be generated by the eyetracking process. The proposed algorithm is a technique for the detection of head nod and shakes gestures based on eye-tracking and head motion decision, whereas real-time head region detection is based on differences in image frames using an active camera. The main components of the system include the application of vertical projection of edge contours to identify head location as well as a nested $\mathrm{K}$-means algorithm for error minimization during differencing and threshold. HMMs absorb some of the errors of eye location, and recognition systems can be effectively applied to a visual user interface.

A fourth paper by Han et al. introduced a method for the implementation of personal healthcare services to support standard personal healthcare devices on an automotive system. They proposed several standards for personal healthcare devices and interfaces for the connection of a personal healthcare device, such as automotive environments that include various network interfaces for infotainment and interconnection between infotainment systems, devices, and platforms. The results showed that not all personal healthcare services were examined, only PAN and simple services in an automotive environment. NFC is very useful for Bluetooth pairing, although more wide service models are required. Research on the implementation of WAN on a V2X network is needed for wide network coverage. For helpful remote services, implementation of HL7, IHL, and W3C on WAN will be included. Further, a more practical service model, which can combine already existing services in an automotive environment, and a theoretical service model for future technology like a CPS are needed.

A fifth paper by Kim et al. presented a cost-effective home energy saving system based on a small embedded system with a remote control feature. For this purpose, they implemented a system that can control LEDs by using an interface board connected to a wireless router ported with OpenWrt by a smartphone. In order to develop an application using wireless routers, a cross-development environment should be utilized. Cross-compiler tools are used to generate executables for an embedded system or multiple platforms. It is used to compile a platform upon which it is not feasible to perform compiling, such as microcontrollers that do not support an operating system. Another application of the results of this paper may include the control of a remote household automation system via the Internet.

A sixth paper by Park et al. introduced a method for reception performance using the downlink transmission method of the LTE system. They proposed a path selection method in multi-hop relay, a signal model, and frame structure, thereby reducing signal attenuation in the shadow area and improving performance of the LTE downlink system. This provides desirable communication services by improving the receiving performance of the whole system while reducing the isolated area in the LTE downlink system as well as enlarging cell coverage of base stations.

A seventh paper by $\mathrm{Ha}$ et al. presented a method for building a relation model, which is a structural model, to identify the effects of introducing RFID into the supply chain of the food and beverage industry. The research framework, based on the literature, consisted of six parts: procurement, production, transport, sale, warehousing, and administration. Exploratory factor analysis (EFA) was conducted in order to determine the factors' structure with observatory variables as well as confirm such variables' reliability and eliminate inadequate observatory variables. The CFA technique is based on comparison of the variance-covariance matrix obtained from the sample to form the model. The introduction of RFID promotes information interchanges between SC activities, which in turn enable the coordination and consolidation of a total SCM. Ultimately, the RFID system not only enables the improvement of SC partners' utilities but also promotes the efficiency of SCM as a whole.

A eighth paper by Hung, et al. presented an optimal collaboration of thin-thick clients and resource allocation in the context of cloud computing. They proposed a novel architecture, taking advantage of the collaboration between thin and thick clients, optimizing data distribution, and utilizing CC resources, so that expected quality-of-service requirements could be met. We also proposed an algorithm to determine an optimal resource allocation strategy to satisfy various service-level agreements. Using carefully implemented simulations, they showed that their proposed method is able to increase the efficiency of resource allocation and utilization with a suitable strategy, as illustrated 
by the minimum number of VMs used, while also managing to retrieve shorter execution times in data distribution, especially big data in cloud computing, compared to other existing approaches.

Acknowledgments This fine collection of papers was achieved by fruitful collaborations. We gratefully acknowledge and express heartfelt thanks to all the authors for their worthy contribution to this special issue. We also would like to thank all the members of the ICITCS Program Committee and anonymous reviewers for their help in identifying the novel papers and for their careful reading of earlier drafts in order to select 8 high quality papers out of 25 submitted papers, 32 percent acceptance rate. Furthermore, we would like to thank Professor Peter Thomas, editor-in-chief of the international research journals Personal and Ubiquitous Computing (PUC), for his valuable remarks and his undeterred help throughout the publication process of this special issue.

\section{References}

1. Weiser M (1991) Computer for the Twenty-First Century, Scientific American, pp 94-104

2. Song CW, Lee D, Chung KY, Rim KW, Lee JH (2013) Interactive middleware architecture for lifelog based context awareness. Multimed Tools Appl. doi:10.1007/s11042-013-1362-7

3. Song CW, Chung KY, Jung JJ, Rim KW, Lee JH (2011) Localized approximation method using inertial compensation in WSNs. Inf Int Interdiscip J 14(11):3591-3600

4. Lee KD, Nam MY, Chung KY, Lee YH, Kang UG (2013) Context and profile based cascade classifier for efficient people detection and safety care system. Multimed Tools Appl 63(1):27-44 